\section{A propósito del modelo de influencia tripartita en los trastornos de la conducta alimentaria}

\author{
Regarding to tripartite influence model \\ in eating
}

\section{Señor Editor:}

Hemos tomado conocimiento de la carta enviada por Zevallos-Delzo y Catacora-Villasante "Influencia de los factores socioculturales en los trastornos de la conducta alimentaria" (Rev Chil Neuro-Psiquiat 2014; 52 (4): 308-309) en la cual se hace una crítica a nuestro artículo "Perfeccionismo e insatisfacción corporal en los trastornos de la conducta alimentaria" (Rev Chil Neuro-Psiquiat 2014; 52 (2): 103-114).

Agradecemos la opinión allí vertida, la que ciertamente resulta de gran utilidad para este equipo de investigación, en orden a responder a la retroalimentación de una audiencia especializada y con capacidad crítica. Pasaremos a entregar algunos puntos complementarios:

En la carta se hace mención, así como en nuestro artículo, del modelo multifactorial denominado Tripartite Influence Model ${ }^{1}$, como una perspectiva de relieve para la comprensión etiopatogénica sociocultural de los trastornos de la conducta alimentaria (TCA). A la luz de este enfoque, se cita al Sociocultural Attitudes Toward Appearance Questionnaire- $4^{2}$ como una herramienta evaluativa válida para la mensura de las dimensiones que componen al modelo antes puntualizado: internalización de los ideales de delgadez y atléticos, y presión por parte de padres, medios de comunicación y pares. Nuestro equipo considera tal propuesta como un aporte enriquecedor, razón por la cual esta orientación comprensiva fue también incluida en nuestro manuscrito. No obstante, el propósito primario fue analizar descriptivamente la evidencia disponible sobre la relación entre el perfeccionismo y la insatisfacción corporal como rasgos nucleares en el curso clínico de los TCA, desde una postura holística, que abarcó no sólo aspectos socioculturales, sino que también genéticos, psicopatológicos, conductuales, cognitivo-perceptuales, evolutivos y clínico-terapéuticos desde la exposición de casos clínicos. Dada la extensión del contenido y pese a que fueron expuestas algunas escalas evaluativas en torno a las variables estudiadas (Inventario de Desórdenes Alimentarios en su primera y segunda versión $^{3,4}$, que en conjunto evalúan motivación por la delgadez, consciencia interoceptiva, bulimia, perfeccionismo, insatisfacción corporal, desconfianza interpersonal, temor a la madurez, sentimientos de ineficacia personal, ansiedad social, ascetismo y regulación impulsiva, la Escala Multidimensional de Perfeccionismo de Frost ${ }^{5,6}$, que mide perfeccionismo auto-orientado y socialmente prescrito, la Escala Multidimensional de Perfeccionismo deHewitt y Flett ${ }^{7}$, el Test de actitudes alimentarias ${ }^{8}$, el Cuestionario de silueta corporal ${ }^{9}$, el Cuestionario de asertividad de Rathus ${ }^{10}$, la Escala de impulsividad de Barratt ${ }^{11}$ y la Escala de alexitimia de Toronto-II ${ }^{12,13}$ ), no fue posible incluir con mayor detalle otros instrumentos que asimismo aparecen como atingentes.

Agradecemos el interés en nuestro trabajo y reconocemos la relevancia de estudiar los TCA desde la visión psicosocial en población latinoamericana con una versión validada para la población objetiva.

\section{Referencias bibliográficas}

1. van den Berg P, Thompson JK, Obremski-Brandon $\mathrm{K}$, Coovert M. The Tripartite Influence model of body image and eating disturbance: A covariance structure modeling investigation testing the mediational role of appearance comparison. J Psychosom Res 2002; 53 (5): 1007-20.

2. Llorente E, Gleaves D, Warren C, Pérez L, Rakhkovskaya L. Translation and Validation of a Spanish Version of the Sociocultural Attitudes Towards Appearance Questionnaire-4 (SATAQ-4). Int J Eat Disord. 2014. [Epub ahead of print].

3. Garner D, Olmstead M, Polivy J. Development and validation of a multidimensional eating disorder inventory for anorexia nervosa and bulimia. Int J Eat Disord 1983; 2: 15-34. 
4. Garner D. Eating disorder inventory-2 manual. Odessa: Psychological Assessment Resources, 1991.

5. Frost R, Heimberg R, Holt C, Mattia J. A comparison of tw omeasures of perfectionism. Personality and Individual Differences 1993; 14 (1): 119-26.

6. Gelabert E, García-Esteve L, Martín-Santos R, Gutiérrez F, Torres A, Subirà S. Psychometric properties of the Spanish version of the Frost Multidimensional Perfectionism Scale in women. Psicothema 2011; 23 (1): 133-9.

7. Hewitt P, Flett G. Multidimensional PerfectionismScale (MPS): Technical manual. Toronto: MultiHealth Systems, 2004.

8. Garner D, Olmstead M, Bohr I, Garfinkel P. The eating attitudes test: Psychometric features and clinical correlates. Psychological Med 1982; 12: 871-8.

9. Cooper P, Taylor M, Cooper Z. The development and validation of the Body Shape Questionnaire. Int J Eat Disord 1987; 6: 485-94.

10. Rathus SA. A 30-item schedule for assessing asser- tive behaviour. Behav Ther 1973; 4: 398-406.

11. Patton J, Stanford M, Barratt E. Factor structure of the Barratt impulsiven essscale. J Clin Psychol 1995; 51 (6): 768-74.

12. Bagby R, Parker J, Taylor G. Thetwenty-item Toronto Alexithymia Scale II. Convergent, discriminant, and concurrent validity. J Psychosom Res 1994; 38 (1): 33-40.

13. Martínez-Sánchez, F. Adaptación española de la escala de Alexitimia de Toronto (TAS-20). Clínica y Salud 1996; 7 (1): 19-32.

Rosa Behar A. Departamento de Psiquiatría, Escuela de Medicina, Universidad de Valparaíso, Valparaíso, Chile. Gloria Gramegna S. Hospital DIPRECA, Santiago, Chile. Marcelo Arancibia M. Escuela de Medicina, Universidad de Valparaíso, Valparaíso, Chile.

Correspondencia:

Rosa Behar A.

Departamento de Psiquiatría. Facultad

de Medicina. Universidad de Valparaíso.

Subida Carvallo 200, Casilla 92-V

Valparaíso.

E-mail: rositabehar@vtr.net 